\title{
The role of anesthesiologists in promoting smoking cessation
}

\author{
John Oyston, MB
}

Received: 23 April 2011/Accepted: 20 June 2011/Published online: 30 June 2011

(C) Canadian Anesthesiologists' Society 2011

In 1854, the pioneering British anesthesiologist, John Snow, contributed to ending a cholera epidemic by having the authorities remove the handle from the Broad Street pump, a major source of contaminated drinking water. In modern times, anesthesiologists also have an opportunity to help reduce the scale of an epidemic by promoting smoking cessation during the perioperative period.

In 1998, 47,581 Canadians died as a consequence of smoking. They died from lung cancer (13,951 deaths), ischemic heart disease ( 9,289 deaths), and chronic airways obstruction $(6,457$ deaths $) .{ }^{1}$ In developed countries, up to $15 \%$ of the total health care budget is spent on treating the effects of smoking. ${ }^{2}$ Smoking is the leading cause of preventable death in Canada. ${ }^{3}$ The tobacco epidemic has been described as "probably the greatest health disaster in human history". ${ }^{4}$ Clearly, smoking is a major public health issue, but why does this matter to anesthesiologists?

We see the problems caused by smoking in the operating room every day. Many of our patients would not need surgery if they had never smoked. The obvious examples are the cases of lung cancer and vascular disease, but the effects of smoking are systemic and pervasive. The relative risk of cataracts in smokers is 3.17 times that of nonsmokers, ${ }^{5}$ and the relative risk for development of bladder cancer is 3.9 times greater for male smokers and 2.4 times greater for female smokers. ${ }^{6}$ Fractures are more common in smokers; the relative risk for hip fractures is up to 1.84 times that of non-smokers. ${ }^{7}$

J. Oyston, MB ( $)$

Department of Anesthesia, The Scarborough Hospital, 3050 Lawrence Ave East, Toronto, ON M1P 2V5, Canada e-mail: john7@oyston.com
Smoking is also associated with a higher incidence of ST depression during anesthesia. ${ }^{8}$ Compared with patients who never smoked, smokers are twice as likely to suffer a myocardial infarction in the thirty days after surgery. They also have an $80 \%$ higher chance of developing pneumonia, a $40 \%$ greater risk of sepsis, and they are $30 \%$ more likely to die. ${ }^{9}$ With regard to risks of postoperative infections and wound healing, Sorensen ${ }^{10}$ found that the incidence of wound infections was $12 \%$ in smokers compared with $2 \%$ in non-smokers. In a retrospective study, Moller concluded that "smoking was the single most important risk factor for the development of postoperative complications". ${ }^{11}$ Our surgical colleagues, especially in plastic and orthopedic surgery, are well aware that smoking reduces the chances of successful surgery, especially in cases of vascular flaps and spinal fusion. The risk of abdominal wall necrosis after flap surgery increases from $1.0 \%$ in non-smokers to $4.3 \%$ in ex-smokers and $7.9 \%$ in current smokers. ${ }^{12}$ After spinal fusion, non-union is twice as common in smokers as in non-smokers. ${ }^{13}$ The adverse effects of smoking on wound healing are long-lasting. The outcome of anterior cruciate ligament repair at one year is worse in smokers, who have more pain, poorer function, and less ability to return to sports than non-smokers. ${ }^{14}$

Perioperative smoking is often seen as a risk factor which is not easily altered, but evidence suggests otherwise. For example, Moller ${ }^{15}$ was able to get $60 \%$ of preoperative patients to stop smoking and another $23 \%$ to reduce their smoking by at least $50 \%$ simply by offering weekly counselling sessions with a nurse and nicotine replacement therapy. The effect on outcome was dramatic; there was an overall decrease in complication rates from $52 \%$ to $18 \%$. There was also a reduction in wound problems from $31 \%$ in the control group to $5 \%$ in the intervention group. On average, the intervention group 
stayed in hospital for two fewer days than the control group, and $94 \%$ of the patient days in the intensive care unit were attributable to the control group.

Seventy percent of smokers would like to attempt to quit smoking within the next six months. ${ }^{16}$ Upcoming surgery can be considered a teachable moment ${ }^{17}$ a time when smokers are forced to interact with a variety of health care workers, when they are aware of their own vulnerability to disease, and when they also have the additional motivation to quit by a specific date. However, we are not adept at ensuring patients are informed of the risks of perioperative smoking and the benefits of quitting before surgery. Fortytwo percent of patients said they were not informed about the effects of preoperative smoking, and $43 \%$ of anesthesiologists don't routinely advise smokers to quit. ${ }^{17}$

When anesthesiologists see patients preoperatively, they are expected to risk stratify and take steps to reduce perioperative risks. In the context of smoking, a minimal intervention would be "Ask, Advise, and Refer". Specifically, ask the patient about his/her smoking history, advise the patient that continuing to smoke increases the risks of surgery, and refer the patient for smoking cessation counselling, such as the Smokers' Helpline. It would be reasonable to consider this a standard of care.

Some patients already stop smoking before surgery. It has been calculated that $8 \%$ of smoking cessation in the USA is related to surgery. ${ }^{17}$ Major cardiac surgery, such as coronary artery bypass grafting, results in 55\% of patients quitting and remaining smoke free for one year, perhaps because these patients receive effective smoking cessation counselling as part of their surgical care pathway. ${ }^{18}$ Less invasive surgery is less likely to result in quitting. Thirteen percent of patients are smoke free after non-cardiac surgery. Coronary angioplasty results in a $25 \%$ smoking cessation rate, and one reason suggested for the higher success rate for coronary artery bypass graft than for angioplasty is because it is more likely to result in smoking cessation. $^{18}$

The Ottawa Model for Smoking Cessation (OMSC) provides some insight into how smoking cessation can be undertaken in a systematic and effective fashion. This inpatient program, which was developed at the University of Ottawa Heart Institute in 2002, ${ }^{16}$ systematically identifies, provides treatment, and offers follow-up to all smokers admitted to participating hospitals. Implementation of the OMSC has led to a $52 \%$ increase in long-term quit rates (29-44\% at six months). This model of care has proven to be clinically effective $(50 \%$ of individuals remaining smoke free after six months) and cost-effective (with a $300 \%$ return on investment by reducing readmission to hospital and saving bed-days). ${ }^{16}$ Smokers who try to quit with the help of best practice counselling and cessation medications, as incorporated into the Ottawa Model, experience double or triple the success rate for quitting long term. In 2006, the program was extended to other hospitals. Today, nearly 70 sites across Canada have implemented the model. An evaluation of the first nine secondary care hospitals to implement the model revealed an $11.1 \%$ increase in long-term cessation rates (18.3$29.4 \%) .{ }^{16}$ This proven and effective model can and should be extended to preoperative patients so they can gain the benefits of smoking cessation before rather than after surgery.

The benefits of at least six weeks of preoperative smoking cessation have been established. ${ }^{19}$ Why then do we continue to allow patients to come to our operating rooms for elective surgery who have not been provided with counselling about the benefits of smoking cessation and the time to reap the benefits? One concern is anecdotal reports and observational studies which suggest that there may be an increased risk of respiratory complications in patients who quit smoking a few days before surgery. In a comprehensive review of the physiological effects of perioperative smoking, Dr. Warner dismissed these concerns stating, "The evidence suggesting an increase in risk during the first weeks of quitting is insufficient to support any recommendation that smokers not strive for preoperative abstinence, even if only for a brief period before surgery." 20

We expect that patients who are at risk of perioperative cardiac or respiratory adverse events to attend a consultation with an internist or undergo selective cardiac investigations as required. As a logical association, it is also reasonable to expect those at-risk patients who smoke to attend at least one preoperative smoking cessation counselling session. Current best evidence suggests that elective surgery should be delayed by at least six weeks after the counselling to allow time for the benefits of smoking cessation, just as we would delay elective surgery for a patient with a respiratory infection, who was taking anticoagulants or who had any other identifiable and remediable risk factor.

It is desirable to implement a systematic approach to preoperative identification of smoking surgical candidates, an approach which includes a proactive program for providing perioperative smoking cessation counselling and an efficient follow-up process to encourage patients to remain smoke free after surgery. Our preoperative assessment units should play a major role in this process. As $19 \%$ of Canadians are smokers, it is possible that up to 250,000 of the 1.3 million operations undertaken every year in Canada are performed on smokers. If just $50 \%$ of these patients were to quit smoking preoperatively, up to 125,000 patients would have reduced perioperative risk factors and the potential to become lifelong non-smokers. People who quit smoking at age 35 may gain six to eight years of life 
expectancy, and even those who quit at age 65 gain one to four years of life expectancy. ${ }^{21}$ In conclusion, if anesthesiologists routinely ensured that all of our patients had the best possible opportunity to stop smoking before elective surgery, we would be following in the footsteps of John Snow and doing our bit to reduce the toll of one of the major epidemics of our time.

\section{Le rôle des anesthésiologistes dans la promotion de l'abandon du tabac}

En 1854, John Snow, l'anesthésiologiste britannique novateur, a aidé à mettre fin à une épidémie de choléra en demandant aux autorités de retirer la poignée de la pompe à eau sur Broad Street, une importante source d'eau potable contaminée. En des temps plus modernes, les anesthésiologistes ont également l'occasion de contribuer à réduire la propagation d'une épidémie en encourageant l'abandon du tabac en période périopératoire.

En 1998, 47581 Canadiens sont morts de conséquences du tabagisme. Ils sont morts de cancers des poumons (13 951 morts), de cardiopathie ischémique (9 289 morts) et d'obstruction chronique des voies aériennes (6 457 morts). ${ }^{1}$ Dans les pays développés, jusqu'à $15 \%$ du budget total alloué à la santé est dépensé pour traiter les effets du tabagisme. ${ }^{2}$ Le tabagisme est la principale cause de décès évitable au Canada. ${ }^{3}$ L'épidémie de tabagisme a été décrite comme «probablement le plus grand désastre en matière de santé de l'histoire de l'Homme ». ${ }^{4}$ Il va sans dire que le tabagisme est un problème de santé publique majeur, mais qu'ont à voir les anesthésiologistes dans tout cela?

Nous voyons les problèmes que provoque le tabagisme en salle d'opération tous les jours. Nombreux sont nos patients qui n'auraient pas eu besoin de chirurgie s'ils n'avaient jamais fumé. Les cas de cancer du poumon et de maladie vasculaire sont les plus évidents, mais le tabagisme exerce ses effets au niveau systémique, et ces effets sont omniprésents. Le risque relatif de cataracte est 3,17 fois plus élevé chez les fumeurs que chez les non-fumeurs, ${ }^{5}$ et le risque relatif de cancer de la vessie est 3,9 fois plus élevé chez les fumeurs et 2,4 fois plus élevé chez les fumeuses que chez les non-fumeurs. ${ }^{6}$ Les fractures sont plus répandues chez les fumeurs; le risque relatif de fracture de la hanche est 1,84 fois plus élevé que celui que court un non-fumeur. ${ }^{7}$ Le tabagisme est également associé à une incidence plus élevée de sous-décalage du segment ST pendant l'anesthésie. ${ }^{8}$ Par rapport aux patients qui n'ont jamais fumé, les fumeurs courent deux fois plus de risques d'avoir un infarctus du myocarde au cours des 30 jours suivant une chirurgie. Ils courent également $80 \%$ plus de risques de manifester une pneumonie, $40 \%$ plus de risques de sepsie, et $30 \%$ plus de risques de décès. ${ }^{9}$ En ce qui touche aux risques d'infections postopératoires et de cicatrisation, Sorensen ${ }^{10}$ a observé que l'incidence d'infection de plaie était de $12 \%$ chez les fumeurs, comparativement à $2 \%$ chez les non-fumeurs. Dans une étude rétrospective, Moller a conclu que « le tabagisme était facteur de risque unique le plus important lié à l'apparition de complications postopératoires ». ${ }^{11}$ Nos collègues chirurgiens, particulièrement en plastie et en chirurgie orthopédique, sont tout à fait conscients que le tabagisme réduit les chances de succès d'une chirurgie, particulièrement dans les cas de lambeau vasculaire et de spondylodèse. Le risque de nécrose de la paroi abdominale après une chirurgie de lambeau augmente également, passant de $1,0 \%$ chez les non-fumeurs à 4,3\% chez les anciens fumeurs et $7,9 \%$ chez les fumeurs actuels. ${ }^{12}$ Après une spondylodèse, l'échec de la fusion est deux fois plus répandu chez les fumeurs que chez les non-fumeurs. ${ }^{13}$ Les effets défavorables du tabagisme sur la cicatrisation sont de longue durée. Le résultat d'une réparation du ligament croisé antérieur à un an est moins bon chez les fumeurs qui souffrent de davantage de douleur, d'un moins bon fonctionnement et d'une capacité moindre de reprendre le sport que les non-fumeurs. ${ }^{14}$

Le tabagisme périopératoire est souvent considéré comme un facteur de risque difficile à modifier, mais certaines données probantes récentes laissent penser qu'il n'en est rien. Par exemple, Moller ${ }^{15}$ est parvenu à inciter $60 \%$ des patients à arrêter de fumer avant l'opération et $23 \%$ ont réduit leur consommation de tabac d'au moins $50 \%$; il leur a simplement offert des consultations hebdomadaires avec une infirmière et un traitement de remplacement de la nicotine. L'effet sur le devenir de ces patients était pour le moins spectaculaire: l'auteur a observé une réduction globale du taux de complications, passant de $52 \%$ à $18 \%$. Il y a également eu une réduction des problèmes de cicatrisation, passant de $31 \%$ dans le groupe témoin à $5 \%$ dans le groupe intervention. En moyenne, le groupe intervention est resté deux jours de moins à l'hôpital que le groupe témoin, et $94 \%$ des jours-patients à l'unité des soins intensifs étaient attribuables au groupe témoin.

Soixante-dix pour cent des fumeurs souhaiteraient essayer d'arrêter de fumer dans les six mois à venir. ${ }^{16}$ Une chirurgie imminente peut être un moment propice à l'apprentissage, ${ }^{17}$ soit un temps où les fumeurs sont obligés d'interagir avec des travailleurs de la santé, pendant lequel ils sont conscients de leur vulnérabilité face à la maladie, et au cours duquel ils ont en plus la motivation d'arrêter avant une date déterminée. Toutefois, nous ne sommes pas très doués pour nous assurer que les patients sont informés des risques du tabagisme périopératoire et des bienfaits liés à 
l'abandon du tabac avant une chirurgie. Quarante-deux pour cent des patients ont déclaré qu'ils ne connaissaient pas les effets du tabagisme préopératoire, et $43 \%$ des anesthésiologistes ne conseillent pas systématiquement à leurs patients fumeurs d'arrêter. ${ }^{17}$

Lorsque les anesthésiologistes voient les patients en clinique préopératoire, leur rôle attendu est de stratifier le risque et de prendre des mesures afin de réduire les risques périopératoires. Dans le cas du tabagisme, une intervention minimale serait de « demander, conseiller et référer ». Plus spécifiquement, demander au patient quels sont ses antécédents de tabagisme, lui expliquer que, s'il continue à fumer, cela augmentera les risques de la chirurgie, et le référer pour une consultation en abandon du tabac, comme par exemple avec la Téléassistance pour fumeurs. Ces quelques mesures peuvent raisonnablement être considérées comme la norme de soins.

Certains patients arrêtent déjà de fumer avant la chirurgie. On a calculé que $8 \%$ des abandons du tabac aux États-Unis étaient liés à une chirurgie. ${ }^{17}$ Les chirurgies cardiaques majeures, telles que les pontages aortocoronariens, entraînent un abandon du tabac chez 55 \% des patients, sans rechute pendant un an. Ces résultats sont peut-être liés au fait que les patients bénéficient de consultations efficaces pour les soutenir dans l'abandon du tabac dans le cadre du processus de soins chirurgicaux. ${ }^{18}$ Les chirurgies moins majeures, en revanche, ont moins de chances d'entraîner un abandon. Treize pour cent des patients cessent de fumer après une chirurgie non cardiaque. Le taux d'abandon du tabac en cas d'angioplastie coronaire est de $25 \%$. L'une des raisons proposées pour expliquer le taux de réussite plus élevé des pontages aortocoronariens que des angioplasties est le fait qu'il est plus probable qu'un pontage entraîne un abandon du tabac. ${ }^{18}$

Le Modèle d'Ottawa pour l'abandon du tabac (MOAT) offre un aperçu des façons de favoriser un abandon du tabagisme de façon méthodique et efficace. Ce programme pour patients hospitalisés, mis au point à l'Institut de cardiologie de l'Université d'Ottawa en $2002^{16}$ identifie systématiquement tous les fumeurs admis dans les hôpitaux participants, leur propose un traitement et leur offre un suivi. La mise en œuvre du MOAT a entrâné une augmentation de $52 \%$ des taux d'abandon à long terme (29-44 \% à six mois). Il a été démontré que ce modèle de soins était efficace d'un point de vue clinique $(50 \%$ des personnes sont toujours non fumeuses après six mois) et rentable (avec un rendement de l'investissement de $300 \%$ grâce à la réduction des réadmissions à l'hôpital et à l'économie en jours-patients). ${ }^{16}$ Les fumeurs qui tentent d'arrêter en s'aidant des consultations et médicaments d'abandon selon les pratiques exemplaires, telles que celles intégrées dans le Modèle d'Ottawa, ont un taux de réussite de deux à trois fois plus élevé d'abandon à long terme. En 2006, le programme a été étendu à d'autres hôpitaux.
Aujourd'hui, près de 70 sites au Canada ont mis en œuvre ce modèle. Une évaluation des neufs premiers hôpitaux de soins secondaires à intégrer ce modèle a révélé une augmentation de $11 \%$ des taux d'abandon du tabac à long terme (18,3-29,4\%). ${ }^{16} \mathrm{Ce}$ modèle éprouvé et efficace peut et devrait être étendu aux patients en clinique préopératoire de façon à ce qu'ils puissent bénéficier des avantages de l'abandon du tabac avant plutôt qu'après une chirurgie.

Les bienfaits d'un abandon du tabac au moins six semaines avant l'opération ont été établis. ${ }^{19}$ Dès lors, pourquoi continuons-nous à permettre aux patients de se présenter dans nos salles d'opération pour des chirurgies non urgentes s'ils n'ont pas reçu de conseils concernant les avantages d'un abandon du tabac et qu'ils n'ont pas eu le temps d'en récolter les bienfaits? Une inquiétude apparait lorsqu'on pense aux rapports anecdotiques et aux études observationnelles qui indiquent qu'il pourrait y avoir un risque accru de complications respiratoires chez les patients qui arrêtent de fumer quelques jours avant une chirurgie. Dans un compte-rendu exhaustif des effets physiologiques du tabagisme périopératoire, le Dr Warner fait fi de ces inquiétudes en déclarant: "Les données probantes suggérant une augmentation du risque pendant les premières semaines d'abandon sont insuffisantes pour appuyer une quelconque recommandation aux fumeurs de ne pas s'efforcer de s'abstenir en période préopératoire, même si ce n'est que pour une brève période avant la chirurgie. $»^{20}$

Nous nous attendons à ce que nos patients qui courent un risque de complications cardiaques ou respiratoires en période périopératoire respectent nos recommandations et consultent également un interniste ou subissent certains examens cardiaques requis. Logiquement, il est également raisonnable de s'attendre à ce que ces patients fumeurs à risque assistent à au moins une consultation préopératoire d'abandon du tabac. Les meilleures données probantes actuelles indiquent que les chirurgies non urgentes devraient être retardées d'au moins six semaines après la consultation de façon à pouvoir profiter des bienfaits de l'abandon du tabac, tout comme nous retarderions une chirurgie non urgente chez un patient souffrant d'une infection respiratoire prenant des anti-coagulants ou qui présenterait tout autre facteur de risque identifiable ou remédiable.

Il faudrait mettre en œuvre une approche systématique pour identifier, en clinique préopératoire, les fumeurs qui se présentent pour une chirurgie; cette approche devrait inclure un programme proactif afin de proposer des consultations périopératoires d'abandon du tabac ainsi qu'un processus de suivi efficace qui encourage les patients à rester non fumeurs après la chirurgie. Nos unités d'évaluation préopératoire devraient jouer un rôle majeur dans ce processus. Étant donné que $19 \%$ des Canadiens 
fument, il est possible que jusqu'à 250000 des 1,3 million d'opérations réalisées chaque année au Canada le sont chez des fumeurs. Si $50 \%$ de ces patients arrêtaient de fumer avant leur opération, jusqu'à 125000 patients auraient réduit leurs facteurs de risque périopératoires et pourraient devenir des non-fumeurs à vie. Les personnes qui cessent de fumer à 35 ans peuvent prolonger leur espérance de vie de six à huit ans, et même celles qui cessent à 65 ans gagnent un à quatre ans d'espérance de vie. ${ }^{21}$ En conclusion, si nous, les anesthésiologistes, faisions systématiquement en sorte que tous nos patients disposent des meilleurs outils pour arrêter de fumer avant une chirurgie non urgente, nous marcherions dans les pas de John Snow et ferions notre part pour réduire le fardeau de l'une des principales épidémies de notre époque.

Acknowledgement The author has received no funding for this work.

Competing interests The author has received no funding for this work. Dr. Oyston coordinated the preparation of patient information documents on smoking cessation, printed with an unrestricted grant from Johnson \& Johnson, Inc.

\section{References}

1. Makomaski Illing EM, Kaiserman MJ. Mortality attributable to tobacco use in Canada and its regions, 1998. Can J Public Health 2004; 95: 38-44.

2. Parrott S, Godfrey C. ABC of smoking cessation. Economics of smoking cessation BMJ 2004; 328: 947-9.

3. Natural Resources Canada. The Atlas of Canada. Smoking. Available from URL: http://atlas.nrcan.gc.ca/auth/english/maps/ health/healthbehaviors/smoking/1 (accessed April 2011).

4. Turan A, Mascha EJ, Roberman D, et al. Smoking and perioperative outcomes. Anesthesiology 2011; 114: 837-46.

5. Christen WG, Manson JE, Seddon JM, et al. A prospective study of cigarette smoking and risk of cataract in men. JAMA 1992; 268: 989-93.
6. Howe GR, Burch JD, Miller AB, et al. Tobacco use, occupation, coffee, various nutrients, and bladder cancer. J Natl Cancer Inst 1980; 64: 701-13.

7. Kanis JA, Johnell $O$, Oden A, et al. Smoking and fracture risk: a meta-analysis. Osteoporos Int 2005; 16: 155-62.

8. Woehlck HJ, Connolly LA, Cinquegrani MP, Dunning MB 3rd, Hoffmann $R G$. Acute smoking increases ST depression in humans during general anesthesia. Anesth Analg 1999; 89: 856-60.

9. Katznelson R, Beattie WS. Perioperative smoking risk. Anesthesiology 2011; 114: 734-6.

10. Sorensen LT, Karlsmark T, Gottrup F. Abstinence from smoking reduces incisional wound infection: a randomized controlled trial. Ann Surg 2003; 238: 1-5.

11. Moller AM, Pedersen T, Villebro N, Munksgaard A. Effect of smoking on early complications after elective orthopaedic surgery. J Bone Joint Surg Br 2003; 85: 178-81.

12. Chang DW, Reece GP, Wang B, et al. Effect of smoking on complications in patients undergoing free TRAM flap breast reconstruction. Plast Reconstr Surg 2000; 105: 2374-80.

13. Glassman SD, Anagnost SC, Parker A, Burke D, Johnson JR, Dimar JR. The effect of cigarette smoking and smoking cessation on spinal fusion. Spine (Phila Pa 1976) 2000; 25: 2608-15.

14. Karim A, Pandit H, Murray J, Wandless F, Thomas NP. Smoking and reconstruction of the anterior cruciate ligament. J Bone Joint Surg Br 2006; 88: 1027-31.

15. Moller AM, Villebro N, Pedersen T, Tфnnesen H. Effect of preoperative smoking intervention on postoperative complications: a randomised clinical trial. Lancet 2002; 359: 114-7.

16. University of Ottawa Heart Institute. Ottawa Model for Smoking Cessation. About OMSC. Available from URL: http://www. ottawamodel.ca/en_about.php (accessed April 2010).

17. Shi Y, Warner DO. Surgery as a teachable moment for smoking cessation. Anesthesiology 2010; 112: 102-7.

18. Crouse JR $3^{\text {rd }}$, Hagaman AP. Smoking cessation in relation to cardiac procedures. Am J Epidemiol 1991; 134: 699-703.

19. Thomsen T, Villebro N, Moller AM. Interventions for preoperative smoking cessation. Cochrane Database Syst Rev 2010; 7: CD002294.

20. Warner DO. Perioperative abstinence from cigarettes: physiologic and clinical consequences. Anesthesiology 2006; 104: 356-67.

21. Taylor DH Jr, Hasselblad V, Henley SJ, Thun MJ, Sloan FA. Benefits of smoking cessation for longevity. Am J Public Health 2002; 92: 990-6. 